\title{
2 \\ Radar-Rainfall Technology Integration into Hydrologic and Hydraulic Modeling Projects
}

\author{
Srini Vallabhaneni, Baxter Vieux and Terry Meeneghan
}

This chapter describes the background and methodology for integrating gauge and radar rainfall measurements into hydraulic and hydrologic modeling projects. Considering the importance of rainfall in modeling physical systems such as watersheds or sewer systems, attention to the accuracy, precision, spatial resolution, and processing protocol is essential for successful project completion. Using rain gauge network to adjust radar rainfall requires assessment of the rain gauge network and historical data that can help ensure accurate and reliable data. Radar is growing in acceptance in the management, monitoring, and design of sewer system improvements. Important aspects of how radar measures rainfall include understanding how measured reflectivity is converted to rainfall rates and then adjusted using rain gauges. Limitations and advantages of incorporating this data source depends on quality control and assurance procedures applied by project personnel and by the operators of the radar. The NEXRAD (WSR-88D) radar deployed in the U.S. is a nationwide system that can be used in urban drainage system applications with appropriate post- and real-time processing. Services are available that provide both real-time products useful in sewer system management and real-time control. Retrospective studies are possible for re-constructing events using radar data from multiple or single radar installations, and for which flow monitored data was collected. Comparison of hydrographs produced using rain gauges versus radar reveals that radar is capable of producing accurate hydrographs. Calibrating hydraulic models to account for infiltration and inflow induced by rainfall is

Vallabhaneni, S., B. Vieux and T. Meeneghan. 2004. "Radar-Rainfall Technology Integration into Hydrologic and Hydraulic Modeling Projects." Journal of Water Management Modeling R220-02. doi: 10.14796/JWMM.R220-02.

(C) CHI 2004 www.chijournal.org ISSN: 2292-6062 (Formerly in Innovative Modeling of Urban Water Systems. ISBN: 0-9683681-9-0) 
accurate when using rainfall that is spatially and temporally representative of rainfall over the specific catchment. Results are presented showing that peak flow, depth, volume agreement, and hydrograph shape are better matched than with the closest gauge. Overall performance during calibration using radar rainfall for 29 events revealed that the median agreement between monitored and modeled flow depths is $12.4 \%$. Using representative rainfall for model calibration helps avoid parameter distortion beyond physically realistic or typical values. This improves reliability and confidence in the model that translates translates to better decision making and accurate definition of infrastructure improvement projects. Guidelines and recommendations are provided to ensure proper integration of radar rainfall.

\subsection{Introduction}

Precipitation data provides time-variable input to hydrologic models and therefore the precision, accuracy, and resolution of this data is of critical importance to the successful model application. Inadequate precipitation data introduces calibration errors, or misrepresents model input, which in turn reduces model accuracy and reliability for simulation of the physical system.

Precipitation data has historically been provided for modeling by rain gauges distributed throughout the study area. The accuracy of precipitation data is typically a function of the equipment, its location, and maintenance. Precision is a function of the type of equipment used. Accuracy and precision are, however, typically much less problematic than resolution when working with precipitation data. It has become widely recognized in recent years that the calibration and application of ever-more precise models, specifically those representing sewer systems, is significantly compromised by limitations on precipitation data resolution. Since it is impractical, if not impossible, to achieve the desired resolution by simply adding additional precipitation gauges, radar technology has emerged as a viable means to enhance the spatial coverage of precipitation gauge data. Application of radar technology has also been proven to identify deficiencies in rain gauge data, which is perceived as a reliable source for the precipitation data.

The radar measurements collected using the National Weather Service's (NWS) NEXRAD (NEXt generation RADar) system provide reliable aerial patterns of rainfall at useful spatial and data resolution; however, they may not provide data representitive of quantitative rainfall reaching the ground surface (i.e. ground capture rates) Rain gauges, if installed and maintained properly, 
provide reliable point estimates of ground capture rates. Combining these two types of rainfall measurement techniques, which is accomplished by calibration or adjustment of radar data using rain gauge measurements. This makes reliable aerial distribution of the rainfall possible.

Vallabhaneni et al. (2002) describe steps that can be taken for quality control and assurance (QA/QC) of rain gauge data to improve the accurate and reliability of the data before combining it with radar rainfall estimates. Assessment of radar facilities described by Vieux et al. (2002) is performed before using data from a particular radar or set of radars. Adjustment of the radar using gauge data helps remove systematic error (called bias). The resulting gauge-adjusted radar rainfall provides high-resolution data that is better than either system alone (Wilson and Brandes, 1979).

The following activities are necessary to properly integrate the radarrainfall technology into model development:

1. Evaluate various radar-rainfall technologies and products

2. Review the adequacy of the existing rain gauge network

3. Review of NEXRAD facilities and data pertaining to study area

4. Develop data processing protocol

5. Process the NEXRAD and rain gauge data

6. Integrate catchment-specific rainfall hyetographs into project GIS

This chapter provides general guidelines on each of the key activities listed above to facilitate integration of radar rainfall technology into hydrologic and hydraulic modeling projects.

\subsection{Evaluation of Radar-rainfall Technologies and Products}

\subsubsection{Radar-rainfall Technology}

Radar rainfall is an estimation of rainfall rates/accumulation derived from radar reflectivity. The NWS NEXRAD is also known as WSR-88D, where the $D$ stands for Doppler, and the 88 represents the prototype year (1988). Fuller (1990) traces the history and use of radar and its role in weather measurement and forecasting. Radar, which stands for radio detection and ranging, is based on radio and subsequent radar developments in the early 1900 s, weather radar applications began in World War II when clouds interfered with efforts to detect enemy aircraft. With appropriate modifications for detecting hydrometeors (liquid and frozen precipitation), radar was applied to weather surveillance. 
Peacetime applications followed with the first tornado warning issued from Tinker Air Force Base, Oklahoma in March 22, 1948. The mid 1960's brought 37 radars situated across the United States. In 1980, the NWS established the NEXRAD Program, which now has 137 plus WSR-88D radars deployed nationwide. The first operational NEXRAD installation was at Twin Lakes, Oklahoma in 1990. Figure 2.1 shows the Twin Lakes NEXRAD (KTLX) facility, which is typical of most U.S. installations that rely on towers to support the antennae covered by a radome to shield the mechanically revolving parabolic dish from wind currents and moisture.

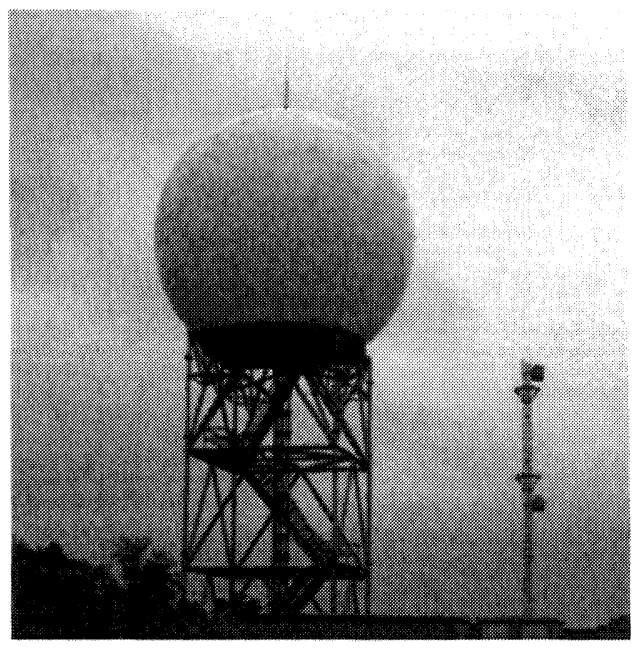

Figure 2.1 Twin Lakes NEXRAD (KTLX) facility tower and radome covering. This first operational NEXRAD is typical of U.S. radar installations.

Figure 2.2 shows precipitation detected by radar over the KILN coverage area, which includes Cincinnati, Ohio. Radar does not measure rainfall directly. The intensity of energy reflected by raindrops, called reflectivity, is converted by the radar system into rainfall estimates through a mathematical relationship called a Z-R relationship. $Z$ represents the reflectivity and $R$ stands for the rain rate The Z-R relationship makes assumptions about the average volume of water in a given volume of atmosphere and includes key factors such as raindrop size and distribution. Supplemented with rain gauge data, radar can provide a valuable source of rainfall information over large areas at high resolution. 


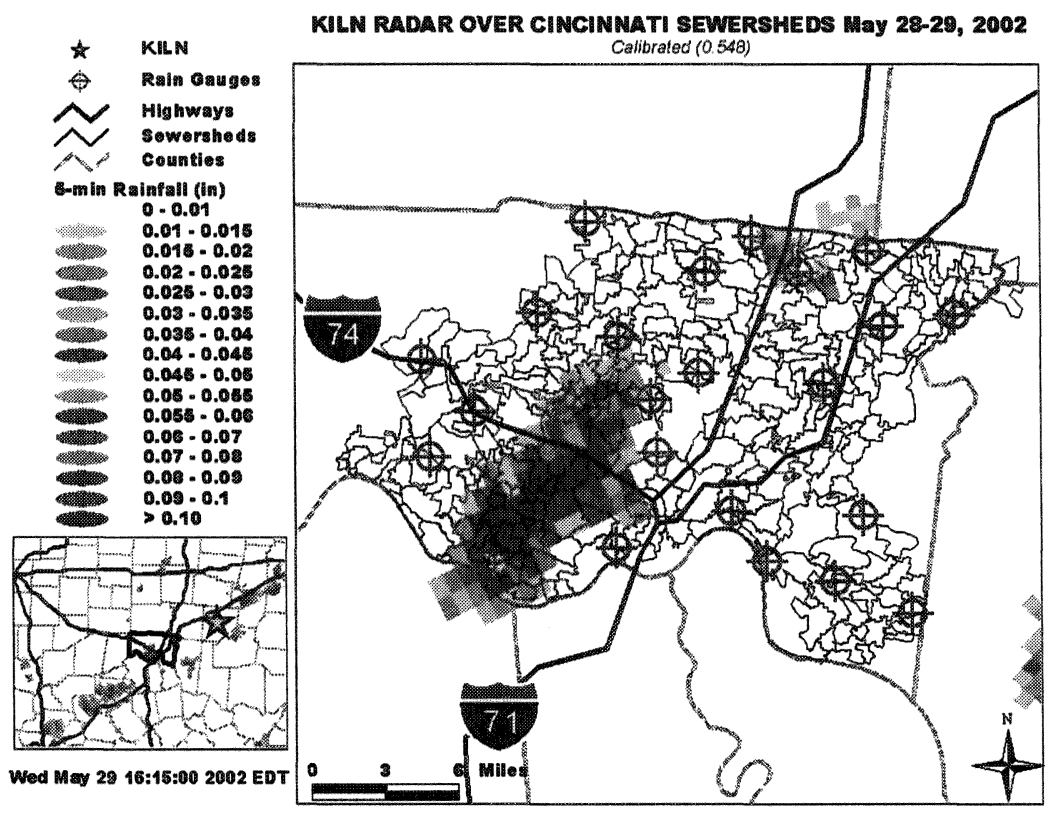

Figure 2.2 KILN radar coverage showing storm precipitation over the 1034 $\mathrm{km}^{2}$ Metropolitan Sewer District of Greater Cincinnati, Ohio (Hamilton County). Rain gauge locations are shown with targets along with radar rainfall depth for the previous 15-minute interval.

At present, the NEXRAD radar network consists of 10-cm (S-band) Doppler radars at over 160 locations in the U.S. and at U.S. military bases worldwide. The operation of the NEXRAD facilities affects the data characteristics resulting from each installation. Distance from the radar facility to the study area is important because of the way in which the radar scans the atmosphere. Starting at $0.5^{\circ}$, with increasing range from the radar, progressively higher elevations are measured. Overshooting low altitude clouds, e.g. stratiform events, may result at far or even intermediate distances. A polar coordinate system is used to sample the atmosphere at intervals of $1-\mathrm{km}$ in range, and 1-degree in azimuth. At $57 \mathrm{~km}$, the width of one radial is approximately $1-\mathrm{km}$, whereas, at $230 \mathrm{~km}$, the width is approximately $4 \mathrm{~km}$.

The NEXRAD radar system covering the U.S. was developed for improved detection of severe weather. Other applications such as sewer system rehabilitation studies and water resources applications have evolved using this 
system. The NEXRAD system produces over one hundred different products used by meteorologists to warn the public about approaching severe weather. Depending on the specific product, additional processing may be required for it to be useful in hydrologic and hydraulic modeling applications. Resolution in space and time, accuracy, and precision are important characteristics that affect its usefulness. Third party processing of NEXRAD measurements is used in real-time and retrospective rainfall applications.

Level II reflectivity (REF) also known as base data contains the full spatial and data precision offered by NEXTRAD. This is 256-level data that is transformed to a rainfall product using an appropriate Z-R relationship and other processing and quality control. NEXRAD level III products include a $4 \times 4-\mathrm{km}$ product that has 256-levels of data precision. It was designed for hydrologic quantitative applications. Level III image products are useful for showing where rainfall is occurring but have only 16 levels of data precision. This low precision means wide intervals between data levels at high rain rates due to the logarithmic scale of reflectivity. Spacing of five decibels of reflectivity means that rainfall rates are known with low precision that becomes less precise with increasing rates. Figure 2.3 shows a graph of the rainfall rate corresponding to each of the intervals contained in the 256- and 16-level products. Data products

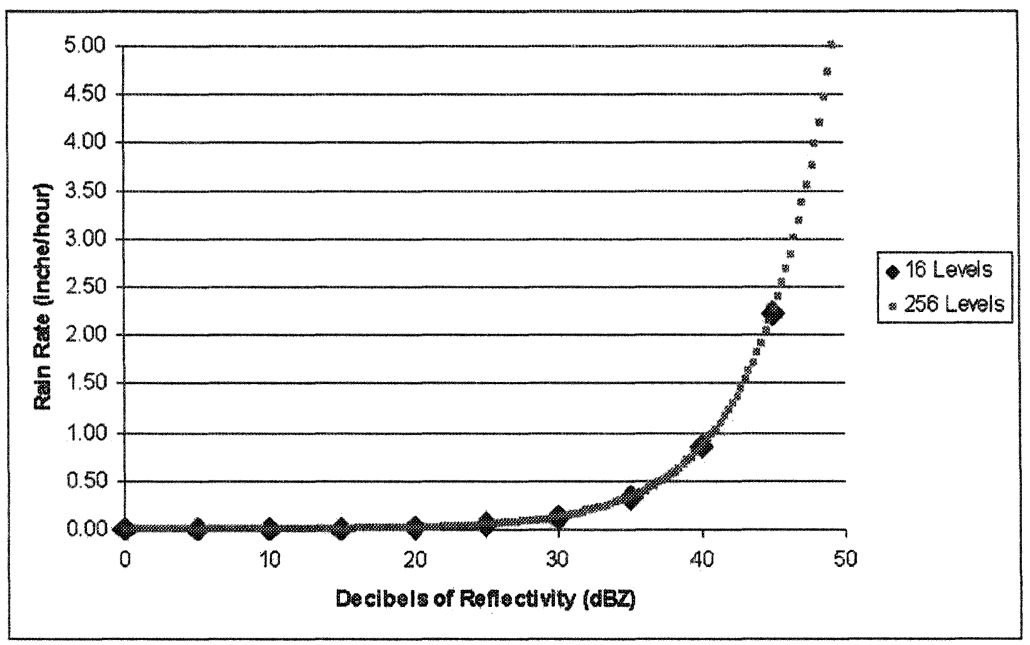

Figure 2.3 Graph of rainfall rate corresponding to each of the intervals contained in the 256- and 16-level products. Finer data precision is evidenced by the number of divisions for the 256-level data. The larger intervals (in excess of $50.8 \mathrm{~mm}$ ) at increasing rainfall rates is typical of low-precision image data on a logarithmic scale. 
contain information for every 0.5 decibels of reflectivity. NEXRAD products that rely on 16-level images have limited value in quantitative applications. Such an image product cannot distinguish rain rates between approximately $0.8 \mathrm{in} /$ $\mathrm{h}(20.3 \mathrm{~mm} / \mathrm{h})$ and $2.2 \mathrm{in} / \mathrm{h}(55.9 \mathrm{~mm} / \mathrm{h})$, or between $2.2 \mathrm{in} / \mathrm{h}(55.9 \mathrm{~mm} / \mathrm{h})$ and $6 \mathrm{in} / \mathrm{h}(152.4 \mathrm{~mm} / \mathrm{h})$. Achieving high accuracy rainfall estimation over a watershed is difficult with low-precision image products. Selecting the bestsuited product for a particular user application is important if expected results are to be achieved. Descriptions of radar applications for hydrology may be found in Bedient and Huber (2001) and Vieux (2001).

Spatial resolution is another data characteristic to be considered. Early development of radar products confronted bandwidth, computational speed, and storage limitations. The polar coordinate system used by NEXRAD measures a reflectivity value for each 1 -degree by $1-\mathrm{km}$ sweep of the radar. This produces more than eighty-two thousand measurements for a radar coverage area. One method used to manage data size is reduction of the data precision. Thus, 16 level images are produced that convey information on where it is raining, etc. For hydrologic applications, a data product called the digital precipitation array (DPA) was devised at $4 \times 4 \mathrm{~km}$. It is not an image product but is a data product with 256 levels of precision that is designed for quantitative applications. Because of its coarser resolution, the number of measurements are reduced from more than eighty-two thousand measurements for a radar coverage area to less than twelve thousand by averaging the polar coordinate radar bins of the native radar measurements while maintaining full data precision. Averaging the individual measurement to a $4 \times 4-\mathrm{km}$ spatial resolution improves computational and file transfer efficiency and reduces anomalous values (outliers). This resolution may be appropriate for larger watersheds where the size is much larger than the resolution of the individual pixels. Figure 2.4 shows the polar coordinate (native resolution) and the DPA $(4 \times 4 \mathrm{~km})$ grid cell. Because of the polar coordinate system, the pixel size grows with distance from the radar. Prior to about 2002, the DPA product was the only 256-level data product available for real-time applications. Subsequently, full precision and resolution (base data) reflectivity was made available in real-time. This was made possible by advances in telecommunication bandwidth and compression technology.

Radar-rainfall data application to urban hydrologic modeling is growing rapidly and is currently used in a number of major cities (e.g. Indianapolis, Pittsburgh, Cincinnati, Seattle, Philadelphia, etc.) In addition, the radar technology and resulting data products are changing at a rapid pace. Technology companies specialize in value-added radar-rainfall services. Both products 


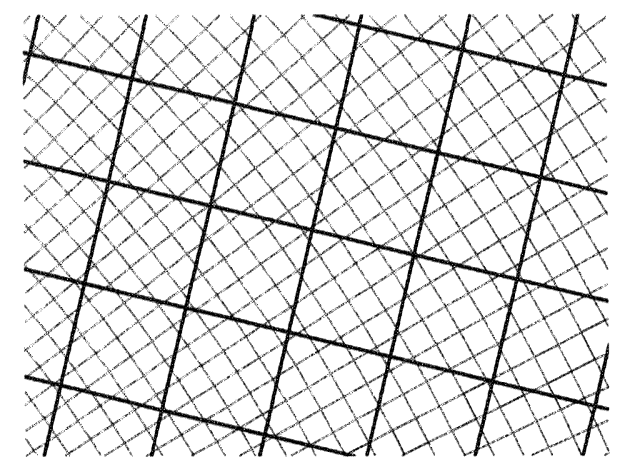

Figure 2.4 Superposition of the $1^{\circ} \times 1 \mathrm{~km}$ polar coordinate (native resolution) and the DPA $(4 \times 4 \mathrm{~km})$ grid cell. In the polar coordinate system, pixel size grows with distance from the radar.

derived from radar (value-added) and NWS products can be purchased directly from vendors with low latency (delay) for real-time applications. The radarrainfall service companies obtain this raw data from NWS and other sources to provide value-added services such as $\mathrm{QA} / \mathrm{QC}$ of the radar and rain gauge data, adjustment of radar data with rain gauge data, GIS formatting, and Internet delivery of gauge-adjusted radar data in desired image and data formats. In general, the data will be provided with a spatial resolution of $1 \times 1-\mathrm{km}$ pixels or coarser, with a time interval of five minutes or more. Graphical images and pixel- and/or catchment-specific time series of radar rainfall are typically provided. Radar rainfall data formats can often be purchased in formats compatible with geographic information system standards such as the ESRI standard Shapefile.

\subsection{Review of Rain Gauge Network}

Assessment of a rain gauge network can help ensure that the data derived from the gauges is of sufficient quality for adjusting the radar data. Quality control and assurance $(\mathrm{QA} / \mathrm{QC})$ of rain gauge data improves the accuracy and reliability of the data. Factors necessary to achieve high quality rain gauge data are described by Vallabhaneni et al. (2002). Rain gauge network assessment reveals chronic and acute conditions affecting gauge data. Review of the spatial distribution of gauges in relation to radar coverage helps identify new gauge 
locations or the need for relocation of existing gauges. Because gauges can become clogged or begin to malfunction, periodic gauge maintenance programs are necessary to maintain data quality and accuracy.

The location and performance of individual rain gauges should be reviewed by qualified personnel. This review should consider items such as spatial distribution, number of gauges, gauge siting, field-measured accuracy, and other relevant factors that impact the radar-rainfall data processing. The review team should also visit each existing rain gauge site to assess and document site conditions (possible obstructions from trees and buildings, wind effects created by surrounding buildings, etc.) that may adversely affect accurate rainfall measurements. For example, Figure 2.5 depicts a site where trees likely cause "rain-shadowing", which should be avoided. Detailed performance testing in the field should be conducted for each rain gauge. Dynamic and static field tests reveal field performance that often differs markedly from laboratory tests. As a result of these field investigations and testing, an assessment of the existing rain gauge network is prepared that provides guidance and a set of recommendations to enhance the network with respect to gauge siting; spatial coverage and number of gauges; and other relevant changes that will support accurate processing of the radar-rainfall data.

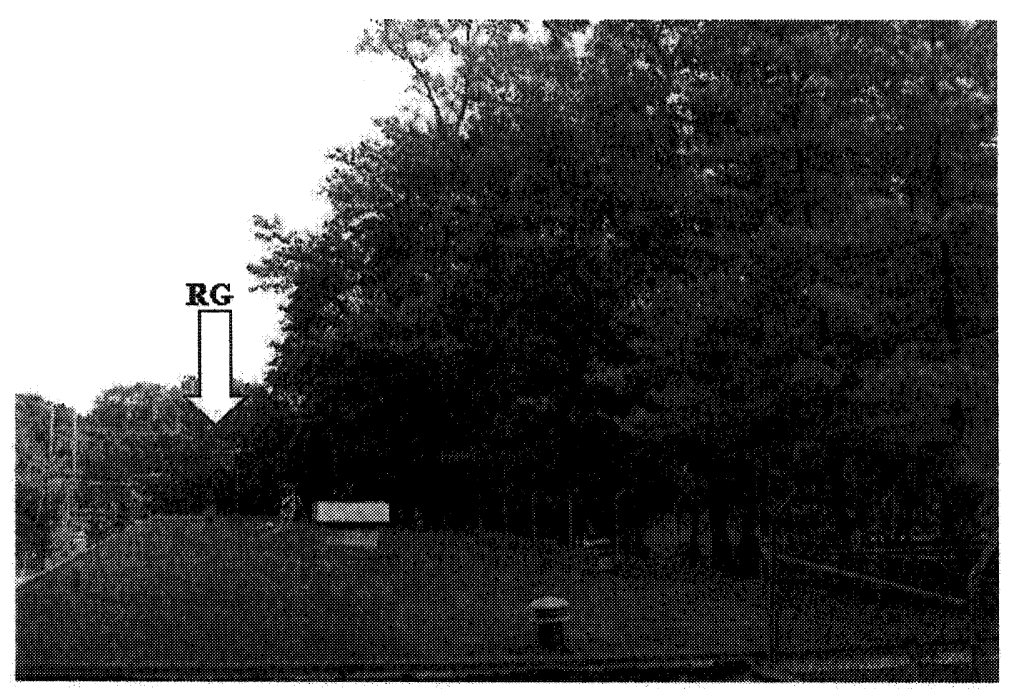

Figure 2.5 Rain shadowed (trees) rain gauge on roof top location (Courtesy of Cincinnati MSD). 
In addition to the field investigations, historical rain gauge data analysis provides insight into the individual gauge operation. A double-mass analysis compares cumulative rainfall totals for two stations or a set of stations with a known standard (typically the nearest NWS station). Double mass analysis is useful for identifying long-term biases or abrupt changes in the data and has long been used to identify shifts in climatic station data (Linsley et al., 1975). When data from a gauge is plotted against the standard gauge data, it should closely follow a 1:1 slope over time. For each unit of rain observed at the standard gauge location, approximately the same depth should be observed at any particular e rain gauge network site. While any individual storm will show some variation due to spatial distance between the gauges, the long-term agreement is remarkably consistent for well-functioning gauges. For this to be true, sufficient observation times are needed. Preferably, one year or a rainy season should be accumulated (preferably at least three months). Some consistent long-term variation is expected climatic differences where mountainous, coastal, or distance influences gauge accumulations. Short-term variation occurs due to spatial variation in rainfall over particular gauges, but does not usually persist over time.

The double mass plot identifies various performance characteristics of the station. Departures of one gauge accumulation from the standard gauge is difficult to determine from the plot alone. However, consistent under- or overreporting due to faulty rain gauge switches, clogged orifices can be identified as potential problems then checked in the field. Gaps in transmission and missing data cause the gauge to report no rainfall while the standard is accumulating rainfall. Rain shadowing at sites often cause variable reductions in accumulations that depend on wind/storm direction in relation to the shadowing feature, e.g. buildings or trees that shelters the gauge from a particular side. Wind eddies caused by buildings or other adjacent features usually reduce rainfall accumulations, but seldom cause increased rainfall accumulations. An example of a double mass plot for one year is shown in Figure 2.6. In this plot the subject gauge agreed well with the standard gauge up to December 2002. Then a period where a clog likely caused under reporting persisted until February (see dotted ellipse). Beyond this period, reasonable agreement with a 1:1 slope (though offset due to the clog) is indicated by the dotted arrow. In summary, the double mass analysis compares accumulations for two stations, identifies long-term biases, gaps, or abrupt changes in the data. Routine maintenance usually corrects these problems. However, event processing for particular time periods benefits from knowing that a particular gauge may have been under-performing. 


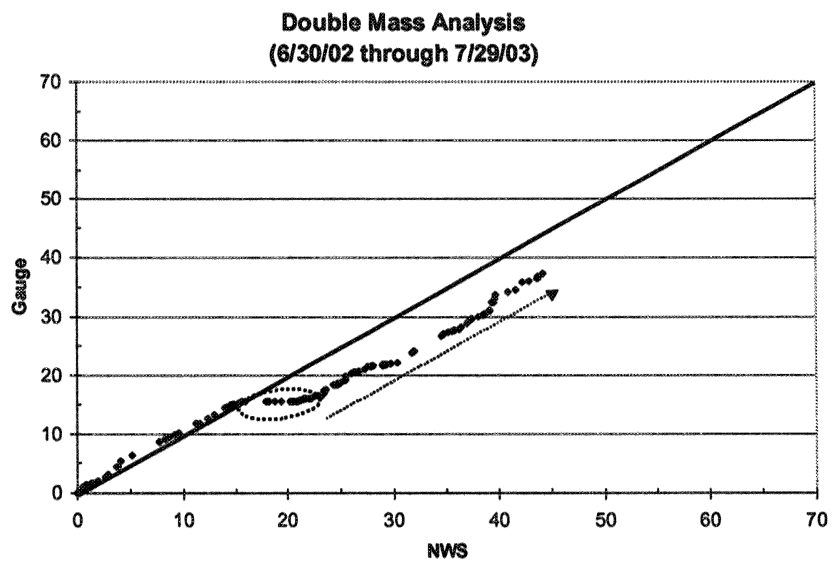

Figure 2.6 Double mass accumulations over one year showing departures of a gauge in comparison to a standard gauge (NWS gauge located within $40 \mathrm{~km}$ ).

\subsection{Review of NEXRAD Facilities for Study Area}

Assessment of radar facilities before using data from a particular radar or set of radars is proven to be beneficial. Operational characteristics of a specific radar installation can have a significant impact on the radar data, and therefore on the radar rainfall data used for hydrologic model calibration. Blockages and power differences may make one radar produce dramatically different results than another. Analysis of a single or multiple radars serving a target area reveals important data quality issues affecting the likely success of a project. Whether radar is being used for a single event analysis, or long-term continuous (annual) support for modeling and system operation, it is worthwhile to review the radar facility. Radar facility analysis based on past events and operational characteristics is described by Vieux et al. (2002). These factors can be just as important as the rain gauge network assessment for identification of characteristics affecting the accuracy of radar rainfall estimates over a particular location or watershed.

The quality of WSR-88D precipitation estimates depends on performance of radar hardware and precipitation algorithms, as well as the algorithm parameters. The radar measures a three-dimensional field of reflectivity, $\mathrm{Z}$, given by:

$$
Z=V^{-1} \sum_{i=1}^{n} D_{i}^{6}
$$


where:

$$
\begin{aligned}
V & =\text { volume of the sample element } \\
n & =\text { number of raindrops in the sample volume, and } \\
D_{i} & =\text { diameter (in millimeters) of the } \mathrm{i}^{\text {th }} \text { drop size. }
\end{aligned}
$$

The drop size distribution changes as the storm evolves and depends on the type of storm event. This distribution affects both the rainfall rate and the reflectivity returned to the radar receiver. The dependence on drop sizes shows the sensitivity of radar to drop sizes. The amount of reflected power by the raindrops, $Z$, is measured and related to rainfall rate, $R(\mathrm{~mm} / \mathrm{h})$, by a $Z-R$ relationship. A typical Z-R relationship used for convective storm events at NEXRAD installations operationally is:

$$
Z=300 R^{1.4}
$$

The particular Z-R relationship applied by the radar operator may change according to season or storm type. The reflectivity factor, $Z$, is usually reported as $10 \log _{10}(Z)$, which is a decibel of reflectance, or $\mathrm{dBZ}$. While Equation 2.2 converts reflectivity to rainfall rate, further adjustment using rain gauge accumulations improves the estimated rainfall accumulations. Consistent under- or over-estimation of rainfall by radar when compared to rain gauges is termed a bias, which is a systematic error removed through adjustment procedures (Vieux, 2001; Bedient and Huber, 2001). Bias removal reduces the systematic error associated with the radar even though random error is unavoidable. Random error is evident when comparing the radar rainfall accumulations over the gauge location, which is due to differences in the spatial scale of the measurements among other factors.

Adjustment to rain gauge accumulations is accomplished after an appropriate or representative Z-R relationship is applied to reflectivity. During a major rainfall event in southeast Texas, October 1994, the WSR-88D radar at Houston-Galveston (KHGX) underestimated the rainfall by as much as $50 \%$ because the standard $Z-R$ relationship $\left(Z=300 R^{1.4}\right)$ used at the time did not represent the drop size distributions of that particular storm event (NWS, 1995). Using the original reflectivity data, Vieux and Bedient(1998) re-analyzed the event with a more appropriate Z-R relationship that better represented the tropical drop size distribution. The tropical $Z-R\left(Z=250 R^{1.2}\right)$ relationship better characterized the October 1994 storm event. Without adjustment of the tropical Z-R, radar accumulations were found to underestimate by $6 \%$ on October 17 , and overestimate by $15 \%$ on October 18 . From this study, the WSR-88D radar was found to be an accurate source of rainfall information provided that an 
appropriate Z-R relationship is used. In most cases, radar rainfall must be adjusted to compensate for the systematic error.

Attenuation of the radar power can occur under extremely heavy precipitation, temporary wetting of the radome, or due to hail cores. The radar consists of both a transmitter and receiver. Deviations in radar transmitter power and receiver losses can cause severe under/over estimation (Doviak and Zrnic, 1993). This is particularly evident when viewing the same event with different radars having different hardware characteristics. Among WSR-88D radars, the relative power is not the same and may change with routine maintenance or parts replacement. Efforts are underway in the U.S. to calibrate the NEXRAD hardware. Even if the power calibration is applied, the power transmitted and received may change as parts are replace or other maintenance is performed. The ratio of the reflectivity and derived rainfall rates should be similar under ideal conditions at equi-distant locations between the radars. Rainfall rates measured at the same location by several radars can be checked from time to time to identify systematic bias caused by power differences.

Procedures for calibrating the WSR-88D hardware, called SuperCal, are described by Chrisman and Chrisman (1999). Hardware calibration is performed by the NEXRAD agencies operating the radar according to agency priority. Deviations in transmitted or received power of just $\pm 2 \mathrm{dBZ}$ can result in rainfall rates that range from $67 \%$ to $150 \%$ of actual rainfall. Thus, rainfall estimation depends heavily on the hardware characteristics of the NEXRAD facilities. Unless careful adjustment is applied, radar power differences will degrade mosaiced radar rainfall products. Power differences affect the quality of radar products produced from a mosaic of individual radars. Once the mosaic of two radars has been performed, it is irreversible making correction of the mosaic difficult if not impossible.

Knowledge gained thorough the NEXRAD facility review is a valuable counterpart to the rain gauge network assessment and should be performed at the outset and periodically thereafter to ensure high quality data.

\subsection{Data Processing Protocol}

For each radar-rainfall application, a data processing protocol should be established. Following a protocol helps maintain uniformity in approach and with consistent accuracy and results. Steps in an example protocol are:

- Describe specific radar products to be used to meet the project goals. 
- Collect radar data. For Level II reflectivity, apply appropriate Z-R relationship.

- QA/QC radar data for anomalous meteorological conditions.

- Develop GIS maps of rainfall.

- Rain gauge QA/QC for missing data or other format problems.

- Compute and remove the mean field bias using valid radar/gauge pairs.

- Compute statistical measures comparing radar and gauge amounts.

- Generate scatter plots of radar/gauge accumulations for storm period.

- Produce basin and point radar hyetographs generated at five-min intervals.

- Documentation for each event should, at minimum, consist of the following:

1. Basin specific hyetographs

2. A scatter plot of the unadjusted radar/gauge pairs of accumulation

3. A scatter plot of the adjusted radar/gauge pairs of accumulation

4. Estimated uncertainty represented by the average difference between gauge and radar

5. Storm total accumulation map and associated table of five-min incremental and storm total amounts.

Details concerning the process of adjusting radar accumulations using gauge data are described below.

\subsection{Radar and Rain Gauge Data Processing}

After QA/QC of the radar and rain gauge data, a critical data processing step is to perform gauge adjustments of radar data using rain gauge network data. Combining rain gauge and radar data to produce improved rainfall estimates is accomplished by a procedure called calibration or adjustment. It is well known that radar can over or under estimate rainfall while providinghigh quality spatial/ temporal patterns of rainfall.

Consistent over/under estimation is the result of systematic error and can be removed given sufficient measurements. As with any measuring device, systematic errors by definition can be removed, while random errors cannot. Comparing gauge and radar estimates of rainfall provides a means of removing 
systematic error known as a bias. An upward bias by $10 \%$ means that the radar is overestimating by $10 \%$. Comparing radar and gauge accumulations for a group of gauges allows computation of a mean field bias. Using the statistics of the radar and gauge pairs, compensation to the radar can be made to remove systematic error. Random error still remains. The average difference characterizes random error between rain gauge and radar accumulations after the bias is removed. Through the process of radar to gauge calibration, a more accurate estimate of rainfall over any particular sewershed can be made.

A mean field bias consists of comparing many radar/gauge pairs of accumulations and then averaging for the mean over the geographic area or window. This geographic window does not have to be limited to the project service area, but can extend to include NWS gauges farther a field. Adjustment of the radar to rain gauge accumulations is the most commonly used technique for correcting radar rainfall estimates.

Wilson and Brandes (1979) described removal of systematic errors in radar estimates using rain gauge accumulations. The mean depth computed overmany gauges provides a statistical basis for removing bias. The multiplicative factor, $\mathrm{F}$, is used for removing the mean field bias. It is defined as the ratio of the mean gauge and radar accumulations:

where:

$$
F=\frac{\frac{1}{n} \sum_{i=1}^{n} G_{i}}{\frac{1}{n} \sum_{i=1}^{n} R_{i}}
$$

$$
\begin{aligned}
G_{i}, R_{i} & =\text { the } \mathrm{i}^{\text {th }} \text { gauge-radar pairs of accumulations, and } \\
n & =\text { the number of pairs. }
\end{aligned}
$$

When $F$ in Equation 2.3 is computed for a storm event total, $G_{i}$ and $R_{i}$ represent total accumulations for the event. This correction may be applied on shorter time intervals such as hourly.

Bias is removed from the radar maps by multiplying cell values in each map by the multiplicative factor $F$. The accurate determination of the random errors is difficult considering that there is a mismatch in space and time between the point values measured by the rain gauge and the areal values measured by the radar. Uncertainty associated with the radar estimates is computed by the average difference between the gauge and radar accumulations after bias removal. Agreement between the gauge and radar pairs is a measure of uncertainty in the resulting rainfall estimates. An example of gauge and radar 


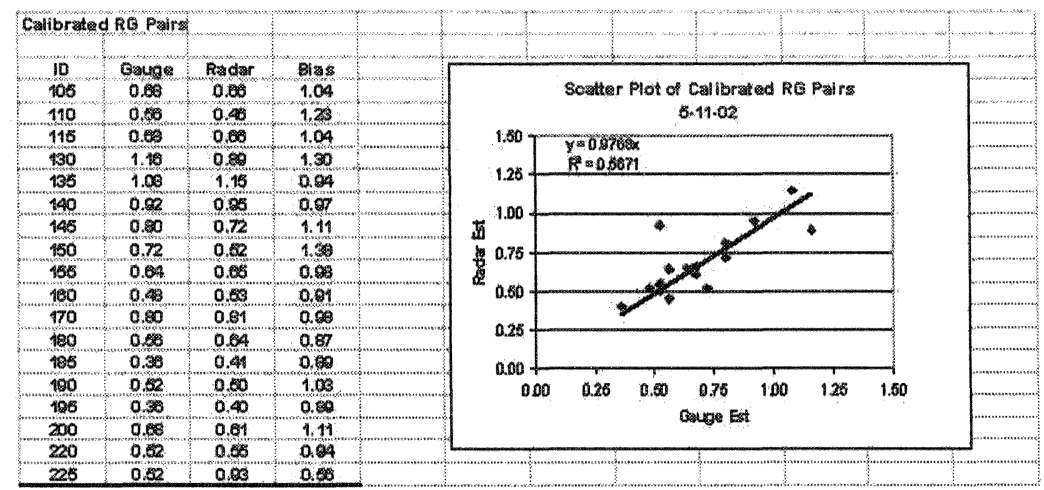

Figure 2.7 Gauge/radar pairs for an event with systematic error removed and remaining random errors. Depth shown in inches $(1 \mathrm{in}=25.4 \mathrm{~mm}$ ). (Courtesy of Cincinnati MSD).

data processing is shown in Figure 2.7. The gauge radar pairs are plotted. The slope of the best-fit trendline is $\sim 1.0$ because the radar data is already corrected. The scatter about the trendline is a measure of the remaining random error. Perfect agreement should not be expected unless the map of radar is warped to match point estimates of rainfall. After adjustment, departures between the $\mathrm{Gi}$ and $\mathrm{Ri}$ depths are reasonable considering the differences in measurement scale (point to area) and local factors affecting rain gauge accumulations (e.g. wind eddies) and radar rainfall rates such as down/up drafts that modify the Z-R relationship during storm evolution.

Evaluation of radar rainfall uncertainty can be performed by withholding rain gauges from calibration and used for validation. However, there are usually insufficient numbers of gauges for validation.

\subsection{Catchment Specific Rainfall Hyetograph Generation}

GIS mapping and routines are essential in developing the catchment specific rainfall hyetographs. GIS setup includes the geo-referencing between the WSR-88D pixel grid system and the GIS mapping for the service area with appropriate basin delineation. Key steps include: extraction of point and basin hyetographs for each mapped basin and subbasins; determination of bias-corrected maps of rainfall for entire service area using the computed mean field bias; and for each basin and subbasin in the service area, extraction of 
hyetographs using adjusted radar maps. The time series of rainfall data is also generated for basins and subbasins to incorporate in to hydrologic and hydraulic models.

Precipitation data is the single most important time-variable input to the hydrologic models and therefore its reliability is of critical importance to successful hydrologic model development, calibration and ultimately application to solve engineering problems. The benefits of using gauge-adjusted radar data have been demonstrated through a number of hydrologic and hydraulic modeling projects throughout the United States and elsewhere. Efforts at controlling sewer discharge using radar information to characterize rainfall over sewered areas and for water resources management are reported by Cluckie et al. (1997); Yuan et al. (1999); Burgess et al. (2003); Meeneghan et al. (2202); Vieux etal. (2002); Vallabhenini et al. (2002); and Wride etal. (2003). Figure 2.8 demonstrates an example of how processed radar-rainfall can be different for a sewershed in an urban area compared to the nearest rain gauge. The lack of agreement for each event is due to the spatial scale of the measurement (point versus areal) and the spatial variability in rainfall depth measured by a gauge some distance from the sewershed. In case of the example sewershed in Figure 2.7, using rain gauge data alone is likely to introduce significant calibration and application errors, which in turn reduces model accuracy and reliability for simulation of the physical system.

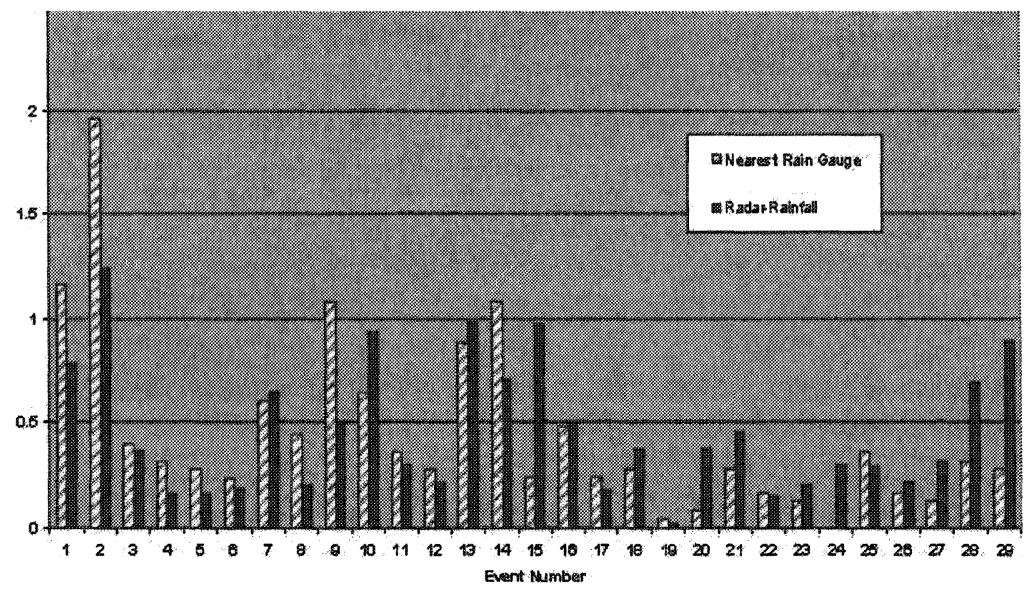

Figure 2.8 Comparison of radar rainfall for a particular sewershed and the nearest rain gauge. Departures indicate spatial variability of rainfall and the non-representative nature of point measures (gauge) compared with areal (radar) rainfall measurements. (Courtesy of Cincinnati MSD). 
Using gauge-adjusted radar over specific sewersheds should produce improved agreement between monitored and simulated flow in the sewer. Comparing hydrographs generated using radar rainfall over the specific catchment with those generated using the closest rain gauge should reveal closer agreement if the radar rainfall is in fact more representative than the gauge. In the course of model calibration, parameter adjustment can compensate for non-representative rainfall. However, this will usually result in distortion of parameters beyond physically realistic or typical values. Model calibration or identification of mis-calibration is not easily done with nonrepresentative rainfall.

Experience shows that achieving reasonable agreement between monitored and simulated hydrographs is easier with radar rainfall. Wride et al. (2003) found better agreement between hydrographs when using catchment-specific radar rainfall than with the closest rain gauge. The median departure between simulated and observed flow depths for the Mill Creek watershed within the Greater Cincinnati Metropolitan Sewer District was found to be $12.4 \%$ over 29 events. Two hydrographs are shown in Figures 2.9 and 2.10 that illustrate the differences in hydrographs obtained using gauge versus radar inputs. The rainfall hyetographs produced by gauge and radar are shown along the upper horizontal axis. In Figure 2.10, the single rainfall pulse measured by the closest gauge causes a delayed and small response compared to that produced by radar. In both events, the gauge introduces considerable timing errors besides a low hydrograph response. To achieve close agreement between observed and simulated flow using the closest rain gauge, considerable distortion in the rainfall infiltration factor would need to be introduced. Even this would not affect the temporal offset introduced by a distant gauge. To avoid parameter distortion, representative rainfall during flow-monitored events is needed. Table 2.1 shows the percent difference between measured and monitored flow volume. The latter quantity is calculated after removal of non-rainfall induced flow (e.g. groundwater contribution). The large disparity shown in the Rain Gauge column (e.g. $-40.2 \%$ ) indicates difficulty in achieving agreement in volume using the closest rain gauge. Lower discrepancies and better volume results are achieved when radar is used, e.g. $-5.4 \%$ during the first event.

During calibration of the System Wide Model for the City of Cincinnati in Mill Creek closer agreement between modeled and monitored results was found when using radar rainfall. This particular model calibration effort relied on 29 events with over 147 flow-monitoring points in 15,480 catchments and 24,451 pipes. The peak flow rates were calibrated to within $12.2 \%$. Peak flow depths calibrated to within $0.19 \mathrm{ft}(5.8 \mathrm{~cm})$ and total flow volume calibrated to within 


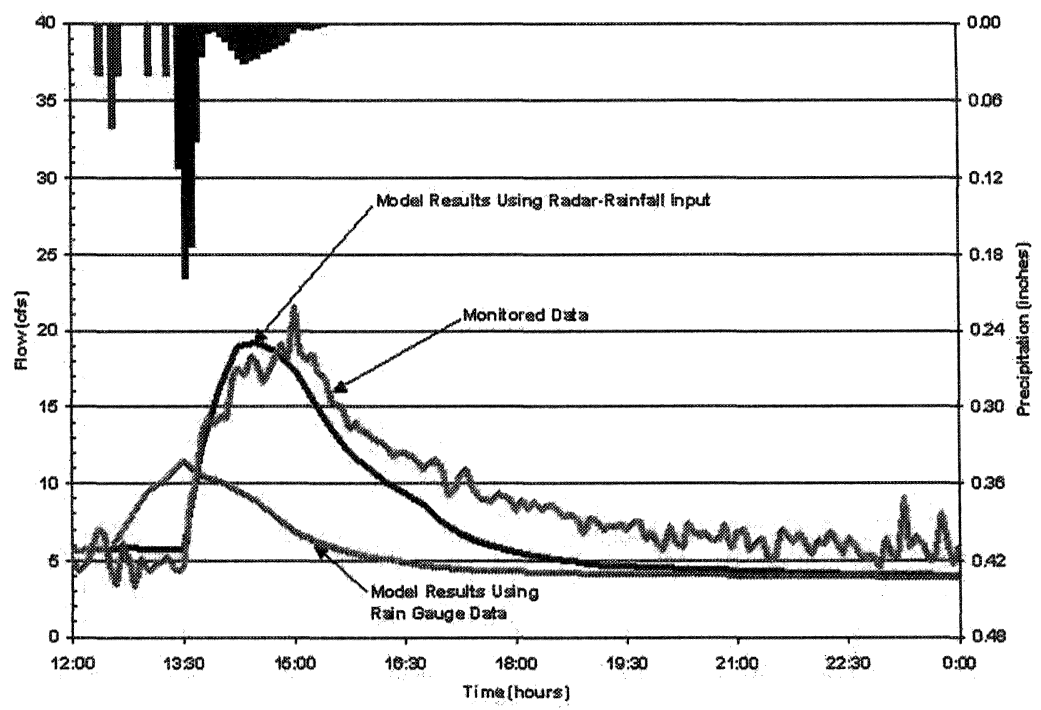

Figure 2.9 Simulated hydrographs derived from radar versus gauge compared with monitored flow. Rainfall pulses at the beginning produce timing and volume errors in the hydrograph simulated using the nearest gauge. Better agreement is produced using radar rainfall over the specific sewershed.

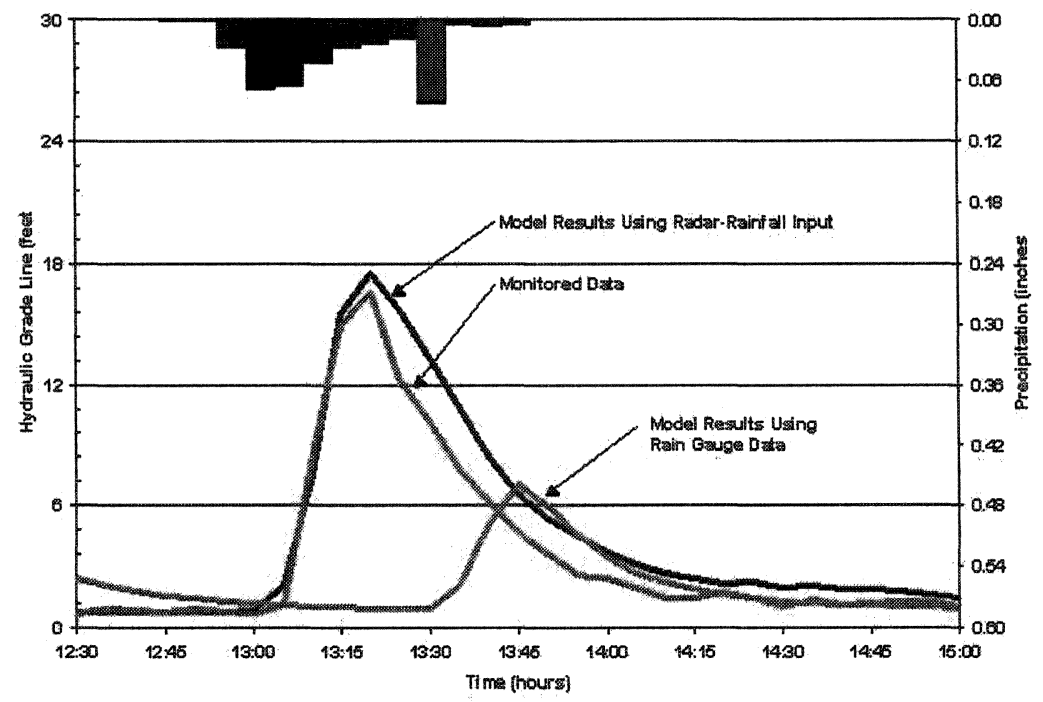

Figure 2.10 Simulated hydrographs derived from radar versus gauge compared with another monitored flow. Radar produces better agreement than hydrographs derived from the nearest gauge. 
Table 2.1 Agreement in simulated and monitored flow volumes derived from radar versus rain gauge.

\begin{tabular}{cccc}
\hline $\begin{array}{c}\text { Mill Creek } \\
\text { Sewershed ID }\end{array}$ & Event Date & $\begin{array}{c}\text { Percent Difference Between Simulated and } \\
\text { Monitored Flow Volume }\end{array}$ \\
\hline & & Radar Rainfall & Rain Gauge \\
\hline WMCOR-001 & 10 April 01 & $-5.4 \%$ & $-40.2 \%$ \\
LMC-002 & 17 May 01 & $-11.2 \%$ & $-38.6 \%$ \\
MC-020 & 19 Feb 02 & $-8.1 \%$ & $-44.0 \%$ \\
\hline
\end{tabular}

$11.9 \%$. Using spatially and temporally representative rainfall derived from radar rainfall helps avoid parameter distortion that results in a more reliable model when used with other (non-calibration) events or long-term simulation. This improved reliability in turn provides more confidence in the model used, and the resulting design of sewer rehabilitation and infrastructure projects.

\subsection{Summary}

This chapter presents essential steps for achieving project goals with accurate rainfall inputs derived from radar and rain gauges. Precipitation input to hydrologic and hydraulic models is the most important factor affecting model performance and reliability. Therefore the precision, accuracy, and resolution of rainfall data is of critical importance to successful model application. Inaccurate or non-representative precipitation data introduces calibration errors, or misrepresents model input, which in turn reduces model accuracy and reliability for simulation of the physical system. The radar measurements provide reliable spatial patterns of rainfall at resolutions consistent with model basin and subbasin delineations. The rain gauges, if installed and maintained properly, provide reliable point estimates of the rainfall reaching the ground surface. Combining rain gauge data with radar provides accurate inputs to hydrologic models. Guidelines and recommendations are provided that help ensure properly integrated radar rainfall technology into hydrologic and hydraulic modeling projects. The model calibration process benefits from using representative rainfall, thus avoiding parameter distortion beyond physically realistic or typical values. Improved reliability and confidence in the model translates to better decision making and accurate definition of infrastructure improvement projects. 


\section{Acknowledgments}

The authors would like to thank the Metropolitan Sewer District of Greater Cincinnati for their support in developing this chapter.

\section{References}

Bedient, P.B., Huber, W.C., (2001) Hydrology and Floodplain Analysis, 3rd Edition, Prentice-Hall, Upper Saddle River, NJ, pp. 763.

Burgess, E., P. Stevens, T. George, S. Vallabhaneni (1997) Use of Radar ImageEnhanced Precipitation Data in Combined Sewer Overflow Modeling, Water Environment Federation, 70th Annual Conference and Exposition, October 18$22,1997$.

Burgess, E.H., B.E. Vieux, S. Mosio, and R.C. Johnstone, 2003. Design storm analysis of sewer system capacity. Proceedings of the Water Environment Federation Annual Conference, WEFTEC'03, Conference on Collection Systems, October 11-15, 2003 Los Angeles, CA.

Cluckie, I.D., D. Han, G.L. Austin, I. Zawadski, 1997. Operational utilization of a high resolution hydrological C-Band radar. In Weather Radar Technology for Water Resources Management, eds. B. Braga and O. Massambani, UNESCO Press: 455466.

Chrisman, J., and C. Chrisman, (1999). An operational guide to WSR-88D reflectivity data quality assurance. WSR-88D Operational Support Facility Paper, 15pp. [Available from WSR-88D Operational Support Facility, 3200 Marshall Ave., Norman, OK 73072.]

Doviak and Zrnic, (1993). Doppler Radar and Weather Observations, 2nd edition, Academic Press, New York, NY. p. 224ff.

Fuller, J.F. Thor's Legions: Weather Support to the U.S. Air Force and Army, 19371987.

Koran, J., S. Vallabhaneni, M. Gatterdam, S. Moisio, E. Burgess (2002). Application of Radar-Rainfall Technology in Combined Sewer Model Development and Calibration, Proceedings, WEFTEC 2002.

Linsley, R. K., M. A. Kohler, and J.L. H. Paulhus, 1975. Hydrology for Engineers, ISBN 0-07-037967-3, McGraw Hill, Inc., USA.

Meeneghan T.J., M.D. Loehlein, R.E. Dickinson, R.D. Myers, T. Prevost, 2002. Impacts of Rainfall Data on Model Refinement in the Greater Pittsburgh Area. Proceedings, 9th International Conference on Urban Drainage, International Joint Committee on Urban Storm Drainage of the IWA and the IAHR, Portland $\mathrm{OR}$, Abstract and paper on CDROM.

Vallabhaneni, S., B.E. Vieux, S. Donovan, and S. Moisio, (2002). Interpretation of Radar and Rain Gauge Measurements for Sewer System Modeling. Proceedings, 
9th International Conference on Urban Drainage, International Joint Committee on Urban Storm Drainage of the IWA and the IAHR, Portland OR, Sept. 8-13, 2002.

Vieux, B.E., S. Vallabhaneni, S. Donovan, and S. Moisio, (2002). An Intercomparison Study of NEXRAD Facility Performance for use in Sewer System Modeling. Proceedings, 9th International Conference on Urban Drainage, International Joint Committee on Urban Storm Drainage of the IWA and the IAHR, Portland OR, Sept. 8-13, 2002.

Vieux, B.E. (2001). Distributed Hydrologic Modeling Using GIS. Kluwer Academic Press, Water Science Technology Series, Vol. 38.

Vieux, B.E., and P.B. Bedient, (1998), "Estimation of Rainfall for Flood Prediction from WSR-88D Reflectivity: A Case Study, 17-18 October 1994." Amer. Meteoro. Soc., J. of Weather and Forecasting, Vol. 13, pp. 126-134.

Yuan, J.M., K.A. Tilford, H.Y. Jiang, and I.D. Cluckie, 1999. Real-time urban drainage system modeling using weather radar rainfall data. J. Phys. Chem.. Earth (B), 24(8): 915-919.

Wilson, J. and Brandes, E. (1979). Radar Measurement of Rainfall - a Summary. Bulletin of the American Meteorological Society, 60(9) pp.1048-1058.

Wride, D., S. Vallabhaneni, M. Chen, R. Johnstone, S. Moisio (2003). Using RadarRainfall to Improve the Characterization of the Inflow/Infiltration (I/I) Response. Proceedings, Water Environment Federation, Collection System Specialty Conference, June 1-4, 2003. 